\title{
PRL-3 promotes cell adhesion by interacting with JAM2 in colon cancer
}

\author{
SHENYI LIAN ${ }^{1}$, LIN MENG $^{1}$, XIAOFANG XING ${ }^{2}$, YONGYONG YANG ${ }^{1}$, LIKE QU $^{1}$ and CHENGCHAO SHOU \\ Departments of ${ }^{1}$ Biochemistry and Molecular Biology, and ${ }^{2}$ Gastrointestinal Translational Research, \\ Key Laboratory of Carcinogenesis and Translational Research, Peking University Cancer Hospital \& Institute, \\ Beijing 100142, P.R. China
}

Received March 19, 2015; Accepted March 8, 2016

DOI: $10.3892 / 01.2016 .4836$

\begin{abstract}
Phosphatase of regenerating liver-3 (PRL-3), also termed PTP4A3, is a metastasis-related protein tyrosine phosphatase. Its expression levels are significantly correlated with the progression and survival of a wide range of malignant tumors. However, the mechanism by which PRL-3 promotes tumor invasion and metastasis is not clear. In the present study, the functions of PRL-3 were systemically analyzed in the key events of metastasis including, motility and adhesion. A cell wounding assay, cell spread assay and cell-matrix adhesion assay were carried out to analyze the cell movement and cell adhesion ability of colon cancer, immunoprecipitation and immunofluorescence assay was confirmed the interaction of PRL-3 and JAM2. It was demonstrated that PRL-3 promoted the motility of Flp-In-293 and LoVo colon cancer cells and increased the distribution of cell skeleton proteins on the cell protrusions. In addition, stably expressing PRL-3 reduced the spreading speed of colon cancer cells and cell adhesion on uncoated, fibronectin-coated and collagen I-coated plates. Mechanistically, junction adhesion molecular 2 (JAM2) was identified as a novel interacting protein of PRL-3. The findings of the present study revealed the roles of PRL-3 in cancer cell motility and adhesion process, and provided information on the possibility of PRL-3 increase cell-cell adhesion by associating with JAM2.
\end{abstract}

\section{Introduction}

Metastasis is considered to be one of the most destructive characteristics of cancer. Though the causes and genetic bases of tumorigenesis vary, the key events required for metastasis are similar for all types of cancer, including the

Correspondence to: Mr. Chengchao Shou, Department of Biochemistry and Molecular Biology, Key Laboratory of Carcinogenesis and Translational Research, Peking University Cancer Hospital \& Institute, 52 Fucheng Road, Beijing 100142, P.R. China

E-mail: scc@bjcancer.org

Key words: PRL-3, cell motility, cell adhesion, JAM2 alteration of adhesion ability, the enhancement of motility and the secretion of proteolytic enzymes to degrade the basement membrane $(1,2)$.

The phosphatase of regenerating liver (PRL) family of protein tyrosine phosphatases (PTPs), including PRL-1, PRL-2, and PRL-3, emerges as potential biomarkers and therapeutic targets for various types of malignancy $(3,4)$. Despite of relatively low expression in normal tissues and untransformed cells, high expression of PRL-3 had been found in a variety of cancer tissues, which correlates with disease progression and survival (5-8). Reports from certain groups highlight the oncogenic role of PRL-3 in promoting cancer metastasis through enhanced cell motility and invasiveness (3). Further investigations have demonstrated that PRL-3 stimulates invasiveness by activating the Rho family of small GTPases and matrix metalloproteinase-2 (MMP-2) $(9,10)$. PRL-3 negatively regulates C-terminal Src kinase (Csk) and PTEN, leading to enhanced activities of Src kinase and PI3K/AKT signaling pathways $(11,12)$. By upregulating the activity of signal transducers and activators of transcription (STAT) pathway and the expression of anti-apoptotic factor Mcl-1, PRL-3 confers therapeutic resistance to small molecule inhibitors. In addition, as a downstream target of the tumor suppressor p53, PRL-3 negatively regulates p53 and PRL-3 modulates cell-cycle progression through the PI3K-AKT pathway (13). Despite of these functions, the role of PRL-3 in other key steps of tumorigenesis in uncertain.

JAM2 (or JAM-B) belongs to the junctional adhesion molecule (JAMs) family, which is composed of 6 immunoglobulin-like members: CAR, ESAM, JAM4, JAM-A, JAM-B and JAM-C $(14,15)$. The majority of research into JAMs focuses on the relationship between differential expression of JAMs and leukocyte movement and redistribution. JAM-B and its family members have been associated with endothelial cell-cell adhesion and leukocyte transmigration through homo/heterophillic interaction. JAM-B stabilizes and recruits JAM-C in the junction complex on the cell-cell contacts through heterophillic interaction (16-18). Two independent groups demonstrated that the JAM-B gene is expressed in three stem cell lines using a DNA microarray method $(18,19)$. The relevance of JAMs within cancer development has rarely been reported (20).

In the present study, the effect of PRL-3 on adhesion and motility in the human embryonic kidney cell line 293 and the 
colon cancer cell line LoVo are systemically analyzed. The molecular role of PRL-3 in cell movement and rearrangement of cell skeleton were investigated as were the effects of overexpression of PRL-3 on cell-matrix cell spread speed and cell-matrix adhesion. To explore the potential mechanism of PRL-3 in cell adhesion and movement, JAM2 was investigated as a new interaction protein of PRL-3. The synergism of PRL-3 and JAM2 promotes cancer cell-endothelial cell adhesion. These results provided an indication that the function of PRL-3 in tumor metastasis may be associated with the junctional adhesion molecules. Blocking the interaction of PRL-3 and JAM2 maybe a new approach to inhibiting metastasis in patients in the future.

\section{Materials and methods}

Cell lines, plasmid and antibody. Flp-In-293 (293) cell line (Invitrogen; Thermo Fisher Scientific, Inc., Carlsbad, CA, USA) and the colon cancer cell line LoVo (American Type Culture Collection, Manassas, VA, USA) were cultured in Dulbecco's modified Eagles medium (DMEM) and Ham's F12 K medium supplemented with $10 \%$ fetal bovine serum (FBS, ThermoFisher Scientific, Inc.), respectively. LoVo cells stably expressing PRL-3 and control cells were previously established (10).

The eukaryon plasmid pDsRED-JAM2 (Clontech Laboratories, Inc., Mountainview, CA, USA) was constructed in our laboratory by inserting full length JAM 2 cDNA into a vector. pEBG-JAM2 and pCDNA-Myc-JAM2 were constructed and saved by our laboratory previously. Monoclonal antibody (3B6) against PRL-3 was prepared as previously reported (21).

In vitro wound healing assay. Cells were seeded onto 6-well plates at a sub-confluent density (5x10\%/well). After $12 \mathrm{~h}$, a line was scraped out of the cell monolayer using a 200- $\mu$ l pipet tip and the width of this wound line was captured using an inverted microscope (ECLIPSE TS100, Nikon, Tokyo, Japan) at a $24 \mathrm{~h}$ interval. The speed of motility of the cells was assessed using the degree of healing of the wound line. The experiment was repeated 3 times independently.

Cell spread assay. Six-well plates were coated with $5 \mu \mathrm{g} /$ well collagen I (Cohesion Technologies Inc., Palo Alto, CA, USA), $1 \mu \mathrm{g} /$ well fibronectin (Sigma-Aldrich Corporation, St. Louis, MO, USA) overnight at $4^{\circ} \mathrm{C}$ or left untreated. Next, the plates were blocked with $2 \%$ bovine serum albumin (BSA; Beijing Solarbio Science \& Technology Co., Ltd., Beijing, China) and washed with phosphate-buffered saline (PBS). Cells were seeded at a density of $5 \times 10^{4}$ cells per well in 6 wells and incubated for $15 \mathrm{~min}$ at $37^{\circ} \mathrm{C}$, then the cell morphology was observed under a light microscope (XDS-300C; Caikon Optical Instrument Co., Ltd., Shanghai, China), the attached cells were counted and the percentage of attached cells was estimated.

Cell-matrix adhesion assay. 24-well plates were coated with $5 \mu \mathrm{g} /$ well collagen I (Cohesion Technologies Inc.), $1 \mu \mathrm{g} / \mathrm{well}$ fibronectin (Sigma-Aldrich) overnight at $4^{\circ} \mathrm{C}$ or left untreated. Next, the plates were blocked with $2 \%$ BSA and washed with PBS. Cells were seeded at the density of $1 \times 10^{4}$ cells per well in 6 wells and incubated for $1,2,3$ or $5 \mathrm{~min}$ at $37^{\circ} \mathrm{C}$. Then the un-attached cells of 3 parallel wells were discarded by gently washing 3 times with PBS. The number of cells that unattached from the wells were evaluated by cytometry (Cellometer Auto T4; Nexcelom Bioscience LLC, Lawrence, MA, USA) and the adhesion rate was expressed as the percentage of the mean amount of washed wells to that of un-washed wells.

Reverse transcription (RT)-polymerase chain reaction (PCR). Ec03 and HmEC cells were cultured and RNA was extracted from cells using Invitrogen Trizol reagent (Thermo Fisher Scientific, Inc.). RT was conducted using a Reverse Transcription System (\#A3500; Promega Corporation, Madison, WI, USA) according to the manufacturer's instructions with the following quantities of reagents: Total RNA, $1 \mu \mathrm{g}$; random primers $(0.5 \mu \mathrm{g} / \mu \mathrm{l}), 1 \mu \mathrm{l}$; oligdT $(2 \mu \mathrm{g} / \mu \mathrm{l}), 1 \mu \mathrm{l}$; dNTPs $(10 \mathrm{mM})$, $1 \mu \mathrm{l} ; 5 \mathrm{X}$ buffer, $4 \mu \mathrm{l}$; RNase inhibitor ( $40 \mathrm{U} / \mathrm{ml}), 0.5 \mu \mathrm{l} ; \mathrm{M}-\mathrm{MLV}$ $(200 \mathrm{U} / \mathrm{ml}), 1 \mu \mathrm{l} ; \mathrm{MgCl}_{2}(25 \mathrm{mM}), 1 \mu \mathrm{l} ;$ and $\mathrm{ddH}_{2} \mathrm{O}$ to a total volume of $20 \mu \mathrm{l}$. For PCR, the reaction mixture consisted of $1 \mu 1$ DNA, $1 \mu 1$ upstream primer, $1 \mu 1$ downstream primer, $12.5 \mu 12$ X PCR Master Mix (Thermo Fisher Scientific, Inc.), and $\mathrm{ddH}_{2} \mathrm{O}$ to a total volume of $25 \mu \mathrm{l}$. Primers were purchased from Sangon Biotech Co., Ltd. (Shanghai, China): JAM2 sense, 5'-AGCAGTAGAGTACCAAGGTGA-3'; JAM2 antisense, 5'-TACGGCTGCTATGATGCCAC-3'; GAPDH sense, 5'-CGGAGTCAACGGATTTGGTCGTAT-3'; and GAPDH antisense, 5'-AGCCTTCTCCATGGTGGTGAAGAC-3'. PCR was performed in an Applied Biosystems 2720 Thermal Cycler (Thermo Fisher Scientific, Inc.) using the following reaction conditions: $95^{\circ} \mathrm{C}$ for $5 \mathrm{~min} ; 29$ cycles of $94^{\circ} \mathrm{C}$ for $30 \mathrm{sec}, 58^{\circ} \mathrm{C}$ for $45 \mathrm{sec}$ and $72^{\circ} \mathrm{C}$ for $40 \mathrm{sec}$; and a final step of $72^{\circ} \mathrm{C}$ for $10 \mathrm{~min}$. Products were stored at $4^{\circ} \mathrm{C}$. PCR products were electrophoretically separated on $0.8 \%$ agarose gels and were visualized using GeneGenius Bio Imaging system (Syngene Bioimaging Private Ltd., Gurgaon, India).

Western blot assay and immunoprecipitation. Cells were seeded (1.5x10 $/$ well) and transfected with pEBG-JAM2, pCDNA-Myc-JAM2 $(4 \mu \mathrm{g})$ and the respective vector for $72 \mathrm{~h}$ using Invitrogen Lipofectamine reagent (Thermo Fisher Scientific, Inc.) according to the manufacturer's instructions; they were then lyzed in lysis buffer $(50 \mathrm{mM}$ Tris-HCl, $\mathrm{pH} 7.5$, $150 \mathrm{mM} \mathrm{NaCl}, 1 \% \mathrm{NP}-40,1 \mathrm{mmol} / 1$ phenylmethylsulfonyl fluoride, $1 \mu \mathrm{g} / \mathrm{ml}$ aprotinin, and $1 \mu \mathrm{g} / \mathrm{ml}$ pepstatin) for $20 \mathrm{~min}$ at $4^{\circ} \mathrm{C}$. The supernatant was collected after centrifugation at $12,000 \times \mathrm{g}$ and subjected to western blotting or immunoprecipitation.

For immunoprecipitation, the supernatant was incubated with a mouse monoclonal anti-Myc antibody $(1 \mu \mathrm{g} / \mathrm{ml}$; \#TA100010; OriGene Technologies, Inc., Rockville, MD, USA). Pre-immune serum was used as control. The precipitates were washed four times with lysis buffer and once with $\mathrm{PBS}$, and eluted in $2 \mathrm{X}$ loading buffer. Protein samples were resolved by sodium dodecyl sulfate polyacrylamide gel electrophoresis and electroblotted onto nitrocellulose membranes (Hybond-C; \#RPN303C; GE Healthcare Life Sciences, Little Chalfont, UK), which were then blocked in 5\% skim milk in PBS-Tween, and probed with the indicated antibodies [mouse monoclonal anti-myc (\#TA100010) or anti-GST (\#TA150102) antibodies (OriGene Technologies, Inc.); final concentration 
A

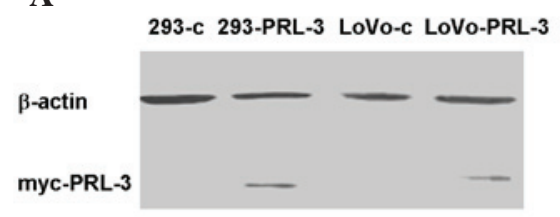

B

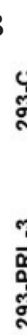

क्ष

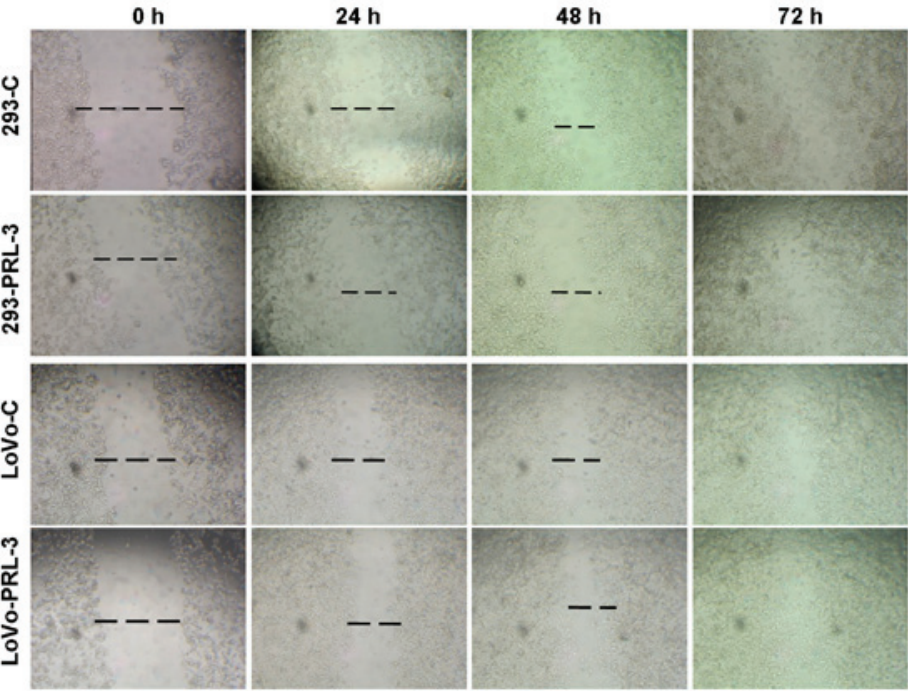

C

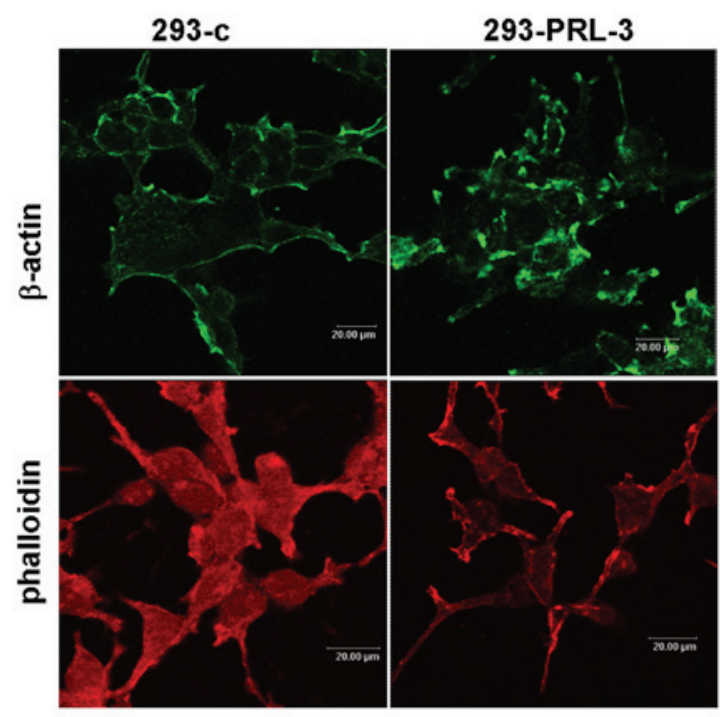

LoVo-c

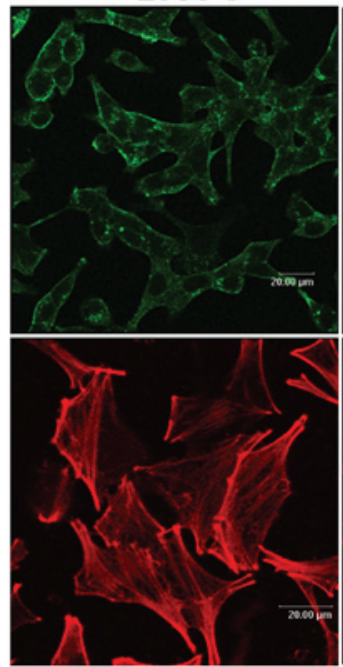

LoVo-PRL-3

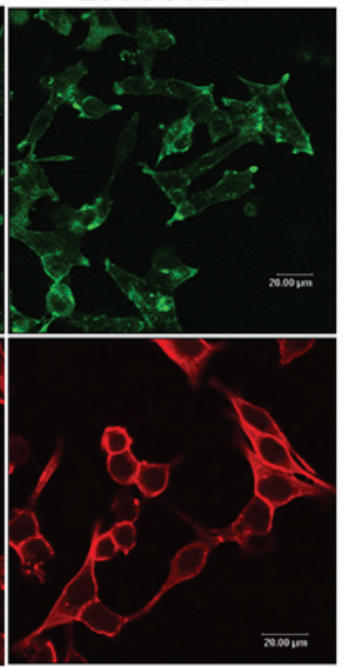

Figure 1. PRL3 promotes colon cancer cell motility. (A) Ectopic PRL-3 expressed in 293 and LoVo cancer cells. $\beta$-actin served as a loading control (magnification, $x 20$ ). (B) PRL-3 promoted cell motility in the cell wound healing assay. (C) Expression of PRL-3 redistributed the cell skeleton protein $\beta$-actin and phalloidin. Scale bar, $20 \mu \mathrm{M}$.

$1 \mu \mathrm{g} / \mathrm{ml}$ ) at $4^{\circ} \mathrm{C}$ overnight, washed with $0.1 \%$ Tween-PBS three times, then incubated with horseradish peroxidase-linked horse anti-mouse IgG antibody (\#7076; Cell Signaling Technology, Inc., Danvers, MA, USA; dilution, 1:2,000) at room temperature for $45 \mathrm{~min}$ ). Protein bands were visualized using a Pierce enhanced chemiluminescence detection system (Thermo Fisher Scientific, Inc.).

Immunofluorescence. LoVo cells were transiently transfected wth pDsRED-JAM2 and pEGFP-PRL-3 plasmid for $48 \mathrm{~h}$, followed by $4 \%$ paraformaldehyde fixation and counterstained with DAPI (1 $\mu \mathrm{g} / \mathrm{ml}$ (\#ZLI-9557; Origene Technologies, Inc.). To label actin filaments, cells were fixed with $4 \%$ paraformaldehyde and stained with $5 \mu \mathrm{g} / \mathrm{ml}$ rhodamine-conjugated phalloidin (Sigma-Aldrich) in the dark for 20 min. Images were captured using a confocal microscope (Lecia TCS SP5; Leica Microsystems GmbH, Wetzlar, Germany) .

Cell-cell adhesion assay. Endothelial cells EC03 and HmEC (China Infrastructure of Cell Line Resources, Beijing, China) were grown on the 24 -well plate $\left(4 \times 10^{5} /\right.$ well) for $24 \mathrm{~h}$, and PBS washed 3 times with gentle shaking, then seeded LoVo cancer cells expressing ectopic PRL-3 and control cell for indicated time point. The cells were carefully washed and non-adhering cancer cells were collected, and counted by hemocytometer. A total of 3 independent experiments were repeated.

\section{Results}

PRL3 promotes colon cancer cell motility. To examine the motility-promoting potential of PRL-3, myc-tagged PRL-3 was stably expressed in 293 and LoVo cells (Fig. 1A). Next, a wounding closure assay was performed. A line was scraped through the cell monolayer and the closure of these lines was recorded at $24 \mathrm{~h}$ intervals. The results demonstrated that the speed of wound healing of 293-PRL-3 and LoVo-PRL-3 were faster than their respective control cells. A total of $48 \mathrm{~h}$ or $72 \mathrm{~h}$ after wounding, the PRL-3 transfected cells had moved to close the wound, while those of their control cells remained apart (Fig. 1B). 
The dynamic regulation of the actin network is crucial for cell motility $(22,23)$. PRL-3 has been reported to regulate the activity of the small GTPase family Rho (11). Rho family members serve an important role in regulating the arrangement of the actin skeleton and pseudopodia. Therefore, the present study examined whether the effect of PRL-3 on motility is related to its role in actin filament remodeling. The distribution of $\beta$-actin by immunofluorescence assay and found that $\beta$-actin was more strongly labeled on the cell protrusions of 293-PRL-3 and LoVo-PRL-3 cells compared to their respective control cells (Fig. 1C), indicating that PRL-3 may participate in the rearrangement of the actin skeleton. The actin filament distribution was stained with rhodamine conjugated-phalloidin, a small molecular toxin that specifically binds to filamentous actin (F-actin), but not monomeric actin. It was observed that F-actin was enriched at the cell membrane, particularly in the protrusion and pseudopodia in 293-PRL-3, while diffusely distributed in 293 control cells. In LoVo cells, F-actin was more strongly labeled in LoVo-PRL-3 cells on the protrusions of the cell membrane compared to distribution of F-actin in LoVo control cells. These data indicated that PRL-3 overexpression may have induced filamentous actin remodeling to promote cell motility.

PRL3 suppresses colon cancer cell spread speed and cell-matrix adhesion. Notably, it was observed PRL-3 reduced the spread speed of colon cancer cells (Fig. 2A). The spreading speed of control and PRL-3 transfected 293 and LoVo cells on extracellular matrix (ECM) components collagen I and fibronectin were examined 15 min after the cells were seeded. Spreading cells appeared as flattened and less refractive, whereas un-spread cells were round and brighter; the percentage of spreading cells to total cells was estimated. As presented in Fig. 2A, 293-PRL-3 and LoVo-PRL-3 cells spread much less than their respective control cells did on un-coated, collagen I-coated or fibronectin-coated plates $(\mathrm{P}<0.05)$. Consistently, PRL-3 expression decreased the cell-matrix adhesion in 293 and LoVo cells at the beginning time point of EDTA-digestion. The unattached cells were counted at the indicated time point following EDTA-treatment, the number of unattached cells of PRL-3 overexpressing group was dramatically higher compared to the control groups. It was concluded that PRL-3 expression promotes cell motility and actin remodeling, and PRL-3 reduces the cell spread and cell-matrix adhesion of cancer cells.

PRL3 interacts with JAM2. To explore the mechanism of PRL-3 in cell adhesion and cell movement, two yeast hybrid systems were used to screen the potential interacting protein(s) of PRL-3 (Fig. 3A). Using BD-PRL-3 fusion protein as a bait protein to screen the embryo brain cDNA librabry, it was demonstrated that JAM2 was a candidate interacting proteins of PRL-3. To confirm the results of the two-yeast hybrid, the interaction between PRL-3 and JAM2 were examined by immunoprecipation with myc-PRL3, followed by western blot analysis with an anti-GST antibody against GST-JAM2, In addition, GST-JAM2 was pulled down and the precipitate was subjected to western blot analysis using an anti-myc antibody against myc-PRL-3 (Fig. 3B). JAM2 is a known protein located on cell membrane, and PRL-3 also locates on cell membrane and plasma. Plasmids encoding pDsred-JAM2 and pEGFP-GFP-PLR-3 were co-transfected into LoVo cells.

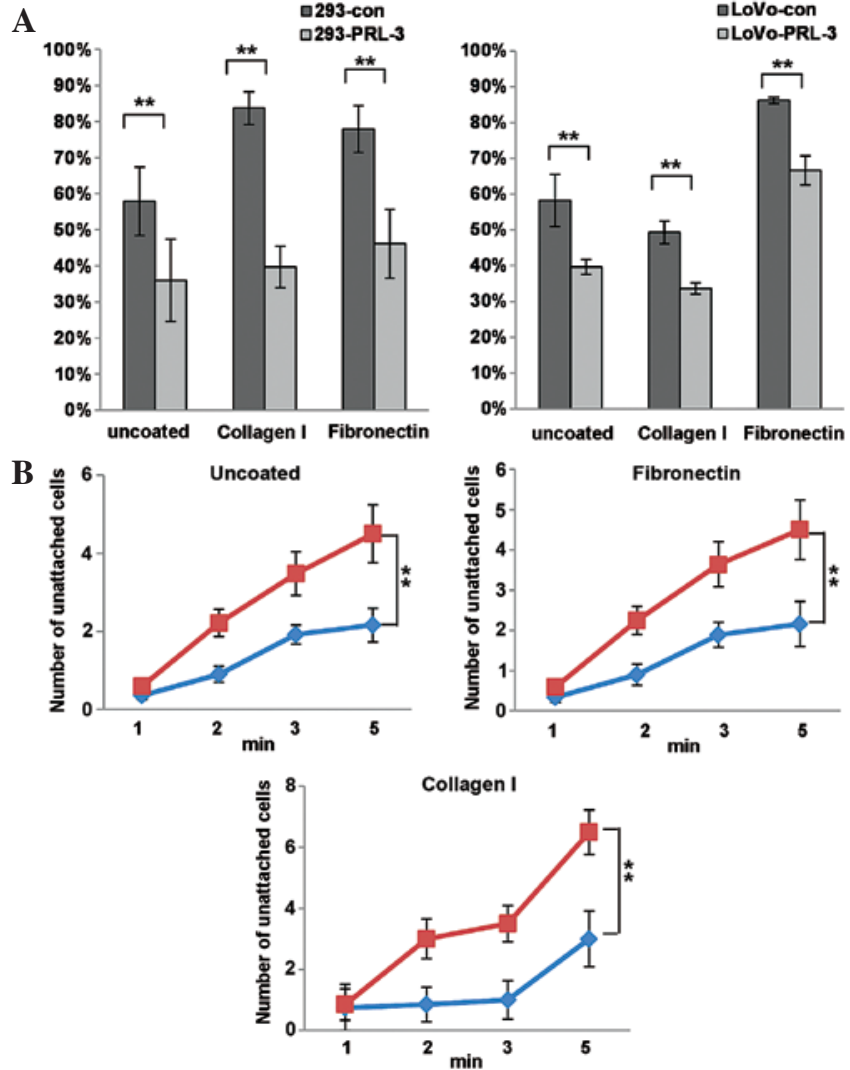

Figure 2. PRL3 suppresses colon cancer cell spread speed and cell-matrix adhesion. The plates were pre-coated with fibronectin or collagen I. (A) Ectopic PRL-3 reduces the cell spread speed in colon cancer cells. ${ }^{* *} \mathrm{P}<0.05$ vs. control cells. (B) Ectopic PRL-3 reduces cell-matrix adhesion. LoVo cells with stably expressed PRL-3 or control cells were seeded on the plates and the unattached cells in the indicated time points were counted. Values are expressed as the mean \pm standard deviation; ${ }^{* *} \mathrm{P}<0.05$ vs. control cells.

After $48 \mathrm{~h}$, the co-localization of exogenous JAM2 and PRL-3 were observed in the cell membrane (Fig. 3C), therefore, PRL-3 may be associated with JAM2 in vitro.

PRL-3 promotes cancer cell-endothelial cell adhesion by associating with JAM2. Cancer metastasis is usually a process in which cancer cells migrate and penetrate the vascular vessels. To investigate this process, different endothelial cell (EC03 and HmEC) were seeded on 96 well plates, and LoVo cells expressing ectopic PRL-3 and control cells were seeded on top and incubated for $15 \mathrm{~min}$. Following this incubation, the cells were washed with PBS 3 times, the number of adhesive cancer cells was estimated by a cytometer. As demonstrated in Fig. 4, the LoVo cells expressing ectopic PRL-3 adhered much more to ECO3 cells compared with the other pairing groups. And reverse transcription-polymerase chain reaction demonstrated that the mRNA expression levels of JAM2 was relatively higher in EC03 cells than in HmEC. These results indicate that ectopic PRL-3 and JAM2 may cooperate to promote cancer cell-endothelial cell adhesion.

\section{Discussion}

Tumor metastasis is a dynamic process involving proliferation of a primary tumor, protrusion of primary lesion, and anchoring 
A

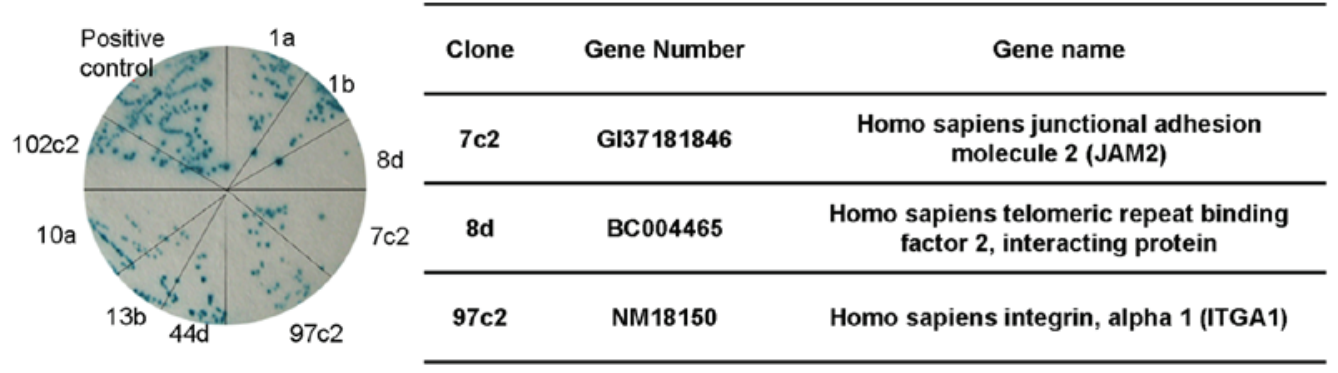

B

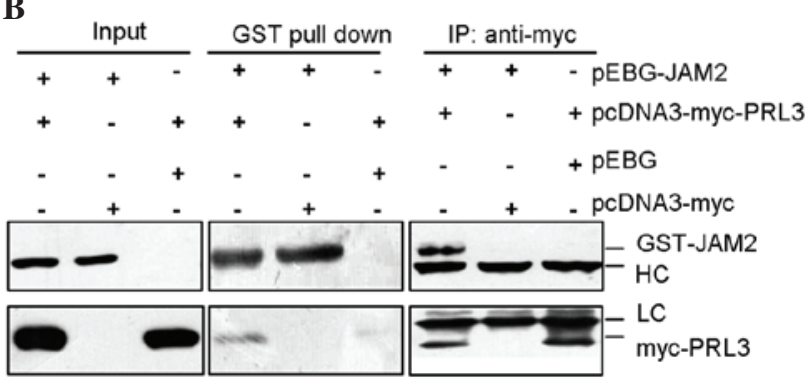

C

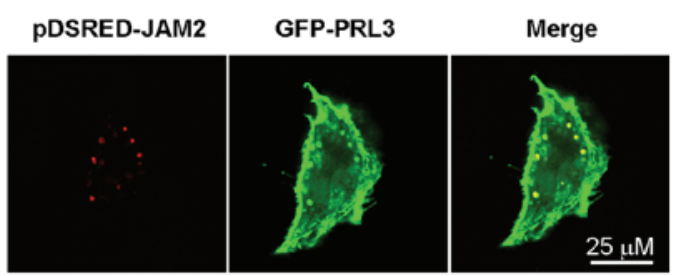

Figure 3. PRL3 interacts with JAM2. (A) The candidate interaction protein of PRL-3 in a yeast two hybrid system. (B) Interaction between PRL-3 and JAM2. Cells were cotransfected with PEBG-JAM2, PcDNA3-myc-PRL-3 plasmids and its control. Protein lysates were immunoprecipitated with an anti-myc antibody or GST antibody. Precipitates and protein were analyzed by western blotting with antibodies against GST and myc. (C) Colocalization of PDSRED-JAM2 and GFP-PRL-3 in LoVo cells. Cells were cotransfected with PDSRED-JAM2 and GFP-PRL-3 vector for 48 h. Scale bar, $25 \mu$ M. JAM2, junctional adhesion molecular 2; IgG H, IgG heavy chain.

onto a secondary site. Cancer cell transport and anchoring on the secondary sites through increased cell-matrix adhesion is a key step for the metastasis $(1,3,26)$. To survive and grow on the secondary sites, cancer cells exhibit more pseudopodia on the cell surface and the cytoskeleton adapts to fasten on the secondary sites (1).

The molecular function of PRL-3 involves its participation in the cancer metastasis process by increasing cancer cell migration and invasion (3). The PI3K-AKT signal pathway is also involved in the process of cell migration and invasion induced by PRL-3. PRL-3 has also been shown to activate EGFR, Src, ERK, JNK, and PI3K-AKT signaling $(3,24,25)$. The role of PRL-3 in the cell movement and cell adhesion is unclear and has not been demonstrated at present.

In the present study, the influence of PRL-3 on the cell motility was observed using a cell wounding healing assay and cell spread assay (Figs. 1B and 2A). One may infer from the results that the stable expression of PRL-3 promotes cancer cell-cancer cell adhesion. Our results showed that PRL-3 promotes cell-cell adhesion and gathering of cancer cells. Although the effect of PRL-3 on the proliferation rate of cancer cells is unclear $(3,27,28)$, the assembled cancer cells with relatively higher expression of PRL-3 have a stronger capability of migration, invasion and autophagy (27). The results indicated that expression of PRL-3 in colon cancer cells aids the survival of primary tumor cells. However, the survival and settlement in the distal organs is the second step in the process of tumor metastasis. Expression of PRL-3 in the colon cancer cells redistributed the cell skeleton protein, forming additional pseudopodia around the cell membrane. The cell matrix binding ability of PRL-3 expressing cells on the uncoated, fibronectin or collagen coated plates were all markedly reduced compared with the control cells. The cancer cells with PRL-3 expression were more easily detached from the coated plates.
$\mathbf{A}$
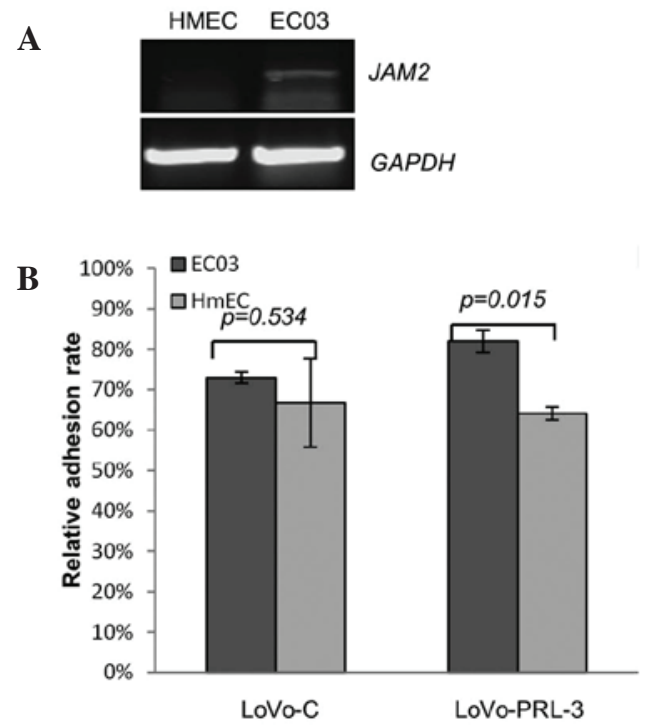

Figure 4. PRL-3 promotes cancer cell-endothelial cell adhesion by associating with JAM2. (A) The mRNA level of JAM2 in the HmEC and EC03 endothelial cells. (B) PRL-3 promotes cancer cell-endothelial cell adhesion by associating with JAM2.

In conclusion, PRL-3 may serve an important function in the process of primary tumor formation and protrusion. To explore the mechanism of PRL-3 in the cell adhesion process, novel interacting proteins of PRL-3 were identified using a yeast hybrid system. Immunopreciptation and GST pull down assay confirmed the interaction between PRL-3 and JAM2.

JAM2 is a protein that associates with tight junctions and enhances homing of lymphocytes to the secondary lymph nodes (15). JAM2 is also an important molecule in the regulation of immune responses and leukocyte migration. 
JAM2 is localized to the cell-cell tight junction and serves a role in the maintenance of endothelial cell architecture (13). As mentioned above, PRL-3 reduces the spread speed and promotes the motility of colon cancer cells (Fig. 1), the present study hypothesiszed that PRL3 expression promotes cancer cells to migrate to secondary sites by increasing cell motility; after homing, PRL3 may promote cancer cell adhesion and invasion on the endothelial cells by associating with JAM2. PRL-3-JAM2 forms the co-localized focal in the cell endomembrane (Fig. 3C). The co-localized focal may aid the PRL-3 expressing cancer cells to anchor and penetrate the vascular endothelial cells. The endothelial cell-cancer cell adhesion assay indicated that PRL-3 expression may increase cell-cell adhesion in the presence of JAM2 expression. Then, the protrusion of primary lesions requires the synergistic action of PRL-3 and JAM2.

Besides the well known function of PRL-3 in the migration and invasion process of colon cancer, the newly identified functions of PRL-3 involve the process of tumor metastasis, particularly the process of cell matrix penetration by tumor cells. Interrupting the interaction between PRL-3 and JAM2 may block the adhesion of vascular endothelial cells and cancer cells. Then the cancer cells may be limited to proliferation in the primary lesions, and the distal metastasis would be reduced. Therefore, the disrupting the interaction between PRL-3 and JAM2 may become a potential target to prevent colon cancer metastasis.

\section{Acknowledgements}

This work was supported by the National Natural Science Foundation of China (grant no. 81301747).

\section{References}

1. Wan L, Pantel K and Kang Y: Tumor metastasis: Moving new biological insights into the clinic. Nat Med 19: 1450-1464, 2013.

2. Maryáš J, Faktor J, Dvořáková M, Struhárová I, Grell P and Bouchal P: Proteomics in investigation of cancer metastasis: Functional and clinical consequences and methodological challenges. Proteomics 14: 426-440, 2014.

3. Al-Aidaroos AQ and Zeng Q: PRL-3 phosphatase and cancer metastasis. J Cell Biochem 111: 1087-1098, 2010.

4. Bessette DC, Qiu D and Pallen CJ: PRL PTPs: Mediators and markers of cancer progression. Cancer Metastasis Rev 27: 231-252, 2008

5. Saha S, Bardelli A, Buckhaults P, Velculescu VE, Rago C, St Croix B, Romans KE, Choti MA, Lengauer C, Kinzler KW and Vogelstein B: A phosphatase associated with metastasis of colorectal cancer. Science 294: 1343-1346, 2001.

6. Xing X, Peng L, Qu L, Ren T, Dong B, Su X and Shou C: Prognostic value of PRL-3 overexpression in early stages of colonic cancer. Histopathology 54: 309-318, 2009.

7. Peng L, Ning J, Meng L and Shou C: The association of the expression level of protein tyrosine phosphatase PRL-3 protein with liver metastasis and prognosis of patients with colorectal cancer. J Cancer Res Clin Oncol 130: 521-526, 2004.

8. Li Z, Zhan W, Wang Z, Zhu B, He Y, Peng J, Cai S and Ma J: Inhibition of PRL-3 gene expression in gastric cancer cell line SGC7901 via microRNA suppressed reduces peritoneal metastasis. Biochem Biophys Res Commun 348: 229-237, 2006.
9. Fiordalisi JJ, Keller PJ and Cox AD: PRL tyrosine phosphatases regulate rho family GTPases to promote invasion and motility. Cancer Res 66: 3153-3161, 2006.

10. Peng L, Xing X, Li W, Qu L, Meng L, Lian S, Jiang B, Wu J and Shou C: PRL-3 promotes the motility, invasion, and metastasis of LoVo colon cancer cells through PRL-3-integrin beta1-ERK1/2 and-MMP2 signaling. Mol Cancer 8: 110, 2009.

11. Liang F, Luo Y, Dong Y, Walls CD, Liang J, Jiang HY, Sanford JR, Wek RC and Zhang ZY: Translational control of C-terminal Src kinase (Csk) expression by PRL3 phosphatase. J Biol Chem 283: 10339-10346, 2008.

12. Wang H, Quah SY, Dong JM, Manser E, Tang JP and Zeng Q: PRL3 down regulates PTEN expression and signals through PI3K promote epithelial-mesenchymal transition. Cancer Res 67: 2922-2926, 2007.

13. Bazzoni G: The JAM family of junctional adhesion molecules. Curr Opin Cell Biol 15: 525-530, 2003.

14. Bradfield PF, Scheiermann C,Nourshargh S, Ody C,Luscinskas FW, Rainger GE, Nash GB, Miljkovic-Licina M, Aurrand-Lions M and Imhof BA: JAM-C regulates unidirectional monocyte transendothelial migration in inflammation. Blood 110: 2545-2555, 2007.

15. Arcangeli ML, Frontera V, Bardin F, Obrados E, Adams S, Chabannon C, Schiff C, Mancini SJ, Adams RH and Aurrand-Lions M: JAM-B regulates maintenance of hematopoietic stem cells in the bone marrow. Blood 118:4609-4619,2011.

16. Doñate C, Ody C, McKee T, Ruault-Jungblut S, Fischer N, Ropraz P, Imhof BA and Matthes T: Homing of human B cells to lymphoid organs and B-cell lymphoma engraftment are controlled by cell adhesion molecule JAM-C. Cancer Res 73: 640-651, 2013.

17. Lamagna C, Meda P, Mandicourt G, Brown J, Gilbert RJ, Jones EY, Kiefer F, Ruga P, Imhof BA and Aurrand-Lions M: Dual interaction of JAM-C with JAM-B and alpha(M)beta2 integrin: Function in junctional complexes and leukocyte adhesion. Mol Biol Cell 16: 4992-5003, 2005.

18. Sakaguchi T, Nishimoto M, Miyagi S, Iwama A, Morita Y, Iwamori N, Nakauchi H, Kiyonari H, Muramatsu $M$ and Okuda A: Putative 'stemness' gene jam-B is not required for maintenance of stem cell state in embryonic, neural, or hematopoietic stem cells. Mol Cell Biol 26: 6557-6570, 2006.

19. Arcangeli ML, Bardin F, Frontera V, Bidaut G, Obrados E, Adams RH, Chabannon C and Aurrand-Lions M: Function of Jam-B/Jam-C interaction in homing and mobilization of human and mouse hematopoietic stem and progenitor cells. Stem Cells 32: 1043-1054, 2014.

20. Morgan C, Jenkins SA, Kynaston HG and Doak SH: The role of adhesion molecules as biomarkers for the aggressive prostate cancer phenotype. PLoS One 8: e81666, 2013.

21. Peng L, Li Y, Meng L and Shou C: Preparation and characterization of monoclonal antibody against protein tyrosine phosphatase PRL-3. Hybrid Hybridomics 23: 23-27, 2004.

22. Fife CM, McCarroll JA and Kavallaris M: Movers and shakers: cell cytoskeleton in cancer metastasis. Br J Pharmacol 171: 5507-5523, 2014

23. Basak S, Jacobs SB, Krieg AJ, Pathak N, Zeng Q, Kaldis P, Giaccia AJ and Attardi LD: The metastasis-associated gene Prl-3 is a p53 target involved in cell-cycle regulation. Mol Cell 30: 303-314, 2008.

24. Rios P, Li X and Köhn M: Molecular mechanisms of the PRL phosphatases. FEBS J 280: 505-524, 2013.

25. Al-Aidaroos AQ, Yuen HF, Guo K, Zhang SD, Chung TH, Chng WJ and Zeng Q: Metastasis-associated PRL-3 induces EGFR activation and addiction in cancer cells. J Clin Invest 123: 3459-3471, 2013.

26. Quail DF and Joyce JA: Microenvironmental regulation of tumor progression and metastasis. Nat Med 11: 1423-1437, 2013.

27. Huang YH, Al-Aidaroos AQ, Yuen HF, Zhang SD, Shen HM, Rozycka E, McCrudden CM, Tergaonkar V, Gupta A, Lin YB, et al: A role of autophagy in PTP4A3-driven cancer progression. Autophagy 10: 1787-1800, 2014.

28. Zhang J, Xiao Z, Lai D, Sun J, He C, Chu Z, Ye H, Chen S and Wang J: miR-21, miR-17 and miR-19a induced by phosphatase of regenerating liver-3 promote the proliferation and metastasis of colon cancer. Br J Cancer 107: 352-359, 2012. 